\title{
A note on the boundary contribution with bad deformation in gauge theory
}

\author{
Bo Feng, Chang-Yong Liu ${ }^{\dagger}$ \\ Center of Mathematical Science, Zhejiang University, Hangzhou, China
}

\begin{abstract}
Motivated by recently progresses in the study of BCFW recursion relation with nonzero boundary contributions for theories with scalars and fermions 1], in this short note we continue the study of boundary contributions of gauge theory with the bad deformation. Unlike cases with scalars or fermions, it is hard to use Feynman diagrams directly to obtain boundary contributions, thus we propose another method based on the $\mathcal{N}=4$ SYM theory. Using this method, we are able to write down a useful onshell recursion relation to calculate boundary contributions from related theories. Our result shows the cut-constructibility of gauge theory even with the bad deformation in some generalized sense.
\end{abstract}

KEYWORDs: BCFW recursion relation, gauge field theory, boundary contribution.

*email address: b.feng@cms.zju.edu.cn

${ }^{\dagger}$ email address: lcy@itp.ac.cn 


\section{Contents}

1. Motivations 1

2. The general framework 3

3. Examples 5

3.1 The first example: gluon MHV amplitude

3.2 The second example: amplitude with two fermions and four gluons $\Lambda_{1}^{+} \Lambda_{2}^{-} g_{3}^{-} g_{4}^{-} g_{5}^{+} g_{6}^{+}$

4. Conclusion and discussions 14

\section{Motivations}

Although generally one can compute any scattering amplitude by Feynman diagrams, as long as the theory has a Lagrangian description, this method is usually complicated in practical applications. In order to make calculations of tree-level and loop-level amplitudes more efficiently, many methods have been suggested in past few decades, among them there is the on-shell BCFW recursion relation [2, 3], which was inspired by Witten's twistor program [4]. Using the BCFW recursion relation, one can construct tree-level amplitudes in terms of sub-amplitudes with fewer external particles, thus it has reduced a big, difficult problem into several smaller and easier ones. The on-shell recursion relation, together with the leading singularities [5], play important roles in recent developments of S-matrix program [6, 7, 8, 9, 10].

The key idea of BCFW recursion relation is to pick up two special momenta $p_{i}, p_{j}$ and do the following deformation (hence we will call it "BCFW deformation") using an auxiliary momentum $q$ :

$$
p_{i}(z)=p_{1}+z q, \quad p_{j}(z)=p_{j}-z q
$$

while other momenta are untouched. With this deformation, the momentum conservation is still kept. Furthermore, the momentum $q$ is chosen to satisfy conditions $q^{2}=0, p_{i} \cdot q=p_{j} \cdot q=0$ so that the deformed $p_{i}(z)$ and $p_{j}(z)$ are kept on-shell. It is only possible for complex $q$ and space-time dimension $D \geq 4$. With the deformed on-shell amplitude $\mathcal{A}(z)$ over single complex variable $z$, we consider following contour integration

$$
\mathcal{B}=\oint_{C} \frac{\mathcal{A}(z)}{z} d z
$$


where contour $C$ is a big enough circle around $z=0$. We can evaluate the integration by two different ways, either by contour around $z=\infty$ or by contour of big circle around the origin. Identified these two results we obtain

$$
\mathcal{A}(z=0)=-\sum_{z_{\alpha}} \operatorname{Res}\left(\frac{\mathcal{A}(z)}{z}\right)+\mathcal{B},
$$

where $\mathcal{A}(z=0)$ is the wanted physical amplitude and $\mathcal{B}$ is the boundary contribution. Residues of poles in the right hand side of (1.3) can always be calculated using factorization properties from lower-point onshell amplitudes. In other words, expression (1.3) shows that for any theory, some parts of tree amplitudes are always "on-shell constructible". Contrast to that, the boundary contribution $\mathcal{B}$ is the obstacle for the application of BCFW recursion relation. If boundary contribution is zero under the chosen BCFW deformation, the whole amplitude is cut-constructible as emphasized in [11]. But for some theories there are no such deformations available to makes $\mathcal{B}$ vanishing, thus we must consider the boundary contribution $\mathcal{B}$ when computing $\mathcal{A}(z=0)$. So it is hard to see if the theory is cut-constructible or not when $\mathcal{B}$ is not zero. Many theories we are familiar with have zero boundary contributions, which usually can not be easily inferred from Feynman diagrams and we should rely on other methods as shown in [12]- [16]. Some theories with non-zero boundary contributions have been discussed in [1], and they are found to be cut-constructible.

For gauge theory, it is well known that one can always find good deformation with zero boundary contribution $\mathcal{B}=0$ [2, 3], such as deformations $\left.\left.\left\langle i^{+}\right| j^{-}\right],\left\langle i^{+}\right| j^{+}\right]$and $\left.\left\langle i^{-}\right| j^{-}\right]$, i.e.,

$$
\lambda_{i}(z)=\lambda_{i}+z \lambda_{j}, \quad \widetilde{\lambda}_{j}(z)=\widetilde{\lambda}_{j}-z \widetilde{\lambda}_{i} .
$$

With these deformations one can calculate tree-level amplitudes $\mathcal{A}$ efficiently. However, there is one bad deformation $\left\langle i^{-}\right| j^{+}$] which does not make the $\mathcal{A}(z)$ vanish at infinity. Although in practise one can always choose a good deformation to simplify calculations and avoid the boundary contributions, it is still important to study the non-zero boundary contributions from the theoretical aspect. Especially we want to ask whether the theory is still cut-constructible with nonzero boundary contributions, and what is the physical implication of these non-zero values.

To calculate non-zero boundary contributions, there are many ways to follow. The first method is to compute them from Feynman diagrams, as have been done in paper [1] for theories with scalars. However, by some simple analysis it is easy to see that this method is not very practical for gauge theory. Unlike theories with scalars and fermions where only a few types of Feynman diagrams give nonzero boundary contributions, there are many types of Feynman diagrams giving potential nonzero boundary contributions in gauge theory, as long as the line from $i$ to $j$ has no more than four four-point vertexes. The second method is to calculate the full amplitude with good deformation and then deform it with bad deformation to get the boundary contributions. This method can get the boundary contributions straightforwardly, but it does not give us the insight whether the boundary part is cut-constructible or not.

In this short note, we propose an alternative method to recursively calculate nonzero boundary contributions with bad deformation for gauge theory and related theory, i.e., the theory with scalars and 
fermions with $\mathcal{N}=4$ interaction terms. The note is organized as following. In section two we will derive an on-shell recursion relation for boundary contributions from the $\mathcal{N}=4$ SYM theory. In section three we will illustrate our idea by two simple examples. One of them is the gluon MHV amplitude and another one, the NMHV amplitude with two fermions. In section four we will give a brief summary of our results.

\section{The general framework}

It is well known that tree-level amplitudes of pure gluons are identical to these obtained from $\mathcal{N}=4$ super-Yang-Mills (SYM) theory. In $\mathcal{N}=4$ SYM theory, one can group all components into following on-shell superfield [17, 4, 18, 19]

$$
\Phi(p, \eta)=G^{+}(p)+\eta^{A} \psi_{A}^{+}+\frac{1}{2} \eta^{A} \eta^{B} S_{A B}+\frac{1}{3 !} \eta^{A} \eta^{B} \eta^{C} \epsilon_{A B C D} \psi^{D-}+\frac{1}{4 !} \eta^{A} \eta^{B} \eta^{C} \eta^{D} \epsilon_{A B C D} G^{-}(p)
$$

with Grassmann coordinate $\eta^{A}, A=1,2,3,4$. Using superfields, amplitudes can be written as functions of $\left(\lambda_{i}, \widetilde{\lambda}_{i}, \eta_{i}^{A}\right)$. For example, the super-MHV amplitude is given by Nair's formula [17] as

$$
\mathcal{A}_{n}(\lambda, \tilde{\lambda}, \eta)=\frac{\delta^{4}\left(\sum_{i} \lambda_{i} \widetilde{\lambda}_{i}\right) \delta^{8}\left(\sum_{i} \lambda_{i} \eta_{i}^{A}\right)}{\langle 1 \mid 2\rangle\langle 2 \mid 3\rangle \ldots\langle n \mid 1\rangle}
$$

To obtain the corresponding scattering amplitudes with various component configurations we just need to expand above expression as the series of $\eta^{A}$.

Having the super-amplitudes, we need also the supersymmetric version of BCFW recursion relation, which is given in [20, 18]. The super-BCFW deformation contains the usual $\langle i| j]$-deformation for two chosen momenta as given by (1.4), as well as the deformation for $\eta$ variables as

$$
\eta_{j}^{A}(z)=\eta_{j}^{A}-z \eta_{i}^{A}
$$

so that the super-energy-momentum conservation is preserved, i.e., $\sum_{t} \lambda_{t} \eta_{t}^{A}$ is invariant under the full super-BCFW deformation. With this in mind the supersymmetric version of BCFW recursion relation can be written as

$\mathcal{A}=\sum_{L, R} \int d^{4} \eta_{P} M_{L}\left(\lambda_{i}\left(z_{P}\right), \lambda_{P}\left(z_{P}\right), \widetilde{\lambda}_{P}\left(z_{P}\right), \eta_{P}\left(z_{P}\right)\right) \frac{1}{P^{2}} M_{R}\left(\widetilde{\lambda}_{j}\left(z_{P}\right), \eta_{j}\left(z_{P}\right), \lambda_{P}\left(z_{P}\right), \widetilde{\lambda}_{P}\left(z_{P}\right), \eta_{P}\left(z_{P}\right)\right)$.

If the deformed super-amplitude $\mathcal{A}^{S U S Y}(z)$ approaches to zero when $z$ goes to infinity ${ }^{1}$, there is no boundary contribution. A nice property of $\mathcal{N}=4$ super-amplitudes is that no matter what helicities of $(i, j)$ are, $\mathcal{A}^{\mathcal{N}=4}(z)$ approaches to zero with $z \rightarrow \infty$, i.e., there is no bad deformation at all for $\mathcal{N}=4$ SYM theory. Having this fact, we expand the super-amplitude as the series of $\eta$ as

$$
\mathcal{A}_{n}=\sum_{a_{1} . . a_{n}=0}^{4} \mathcal{A}_{a_{1} \ldots a_{n}} \prod_{i=1}^{n} \eta_{i}^{a_{i}}
$$

\footnotetext{
${ }^{1}$ It will be interesting to see if there is supersymmetric theory with nonzero boundary contributions for any super-BCFWdeformation.
} 
where $\mathcal{A}_{a_{1} \ldots a_{n}}$ denotes amplitude of field configurations specified by $\left(a_{1}, a_{2}, \ldots, a_{n}\right)$. Under the deformation (1.4) and (2.3) we have

$$
\mathcal{A}_{n}(z)=\sum_{a_{1} . . a_{n}=0}^{4} \mathcal{A}_{a_{1} \ldots a_{n}}(z) \prod_{i=1}^{n} \eta_{i}(z)^{a_{i}}
$$

where $\eta_{j}(z)=\eta_{j}-z \eta_{i}$ and $\eta_{k}(z)=\eta_{k}$ for all the $k \neq j . \mathcal{A}_{a_{1} \ldots a_{n}}(z)$ is the super-amplitude of specific field configuration $\left(a_{1}, a_{2}, \ldots, a_{n}\right)$ after $\left.\langle i| j\right]$-deformation. However, it is more suitable to consider the boundary behavior using the form (2.5) since each super-amplitude of specific configurations is independent in form (2.5). To re-write the deformed super-amplitude expansion (2.6) into the form (2.5), we expand $\eta_{j}(z)$ in (2.6) as follows ${ }^{2}$

$$
\begin{aligned}
& \sum_{a_{1} . . a_{n}=0}^{4} \mathcal{A}_{a_{1} \ldots a_{n}}(z) \eta_{i}^{a_{i}}\left(\eta_{j}-z \eta_{i}\right)^{a_{j}} \prod_{k=1, k \neq i, j}^{n} \eta_{k}^{a_{k}} \\
= & \sum_{a_{1} . . a_{n}=0}^{4} \mathcal{A}_{a_{1} \ldots a_{n}}(z)\left(\sum_{t=0}^{a_{j}} \alpha_{t} \eta_{i}^{a_{i}+t}(-z)^{t} \eta_{j}^{a_{j}-t}\right) \prod_{k=1, k \neq i, j}^{n} \eta_{k}^{a_{k}} \\
= & \sum_{a_{1} . . a_{n}=0}^{4} \eta_{i}^{a_{i}} \eta_{j}^{a_{j}} \prod_{k=1, k \neq i, j}^{n} \eta_{k}^{a_{k}}\left[\sum_{t \geq 0,4 \geq a_{i}+t, a_{j}-t \geq 0} \alpha_{t}(-z)^{t} \mathcal{A}_{a_{i}+t, a_{j}-t}(z)\right]
\end{aligned}
$$

where $\alpha_{t}$ represents the binomial coefficient. After above expansion, we can now explain our method. For the left hand side of (2.6) we have

$$
\oint d z \frac{\mathcal{A}_{n}(z)}{z}=0
$$

and using the fact that each term in the form (2.5) is independent, we get immediately

$$
\oint \frac{d z}{z} \sum_{t \geq 0,4 \geq a_{i}+t, a_{j}-t \geq 0} \alpha_{t}(-z)^{t} \mathcal{A}_{a_{1}, \ldots, a_{i}+t, \ldots, a_{j}-t, \ldots, a_{n}}(z)=0 .
$$

Equations (2.8) gives relations among amplitudes whose field configuration differences from each other only at the positions $i$ and $j$. The boundary contributions of $\mathcal{A}_{a_{i}, a_{j}}(z)$ after $\left.\langle i| j\right]$-deformation are calculated by the following contour integration

$$
\mathcal{B}_{a_{i}, a_{j}}=\oint_{C} \frac{\mathcal{A}_{a_{i}, a_{j}}(z)}{z} d z
$$

where the contour is a big enough circle around $z=0$. Thus one can use the relation (2.8) to calculate boundary contributions of certain amplitude through pole contributions of other amplitudes. For example,

\footnotetext{
${ }^{2}$ Since it is the expansion of Grassman variables, we need to be careful with the sign when changing two variables, which we have not been very careful in our arguments.
} 
the boundary contribution of $\mathcal{A}_{a_{i}, a_{j}}(z)$ is given by

$$
\mathcal{B}_{a_{i}, a_{j}}=\oint_{C} \frac{\mathcal{A}_{a_{1}, \ldots, a_{i}, \ldots, a_{j}, \ldots, a_{n}}(z)}{z} d z=-\oint \frac{d z}{z} \sum_{t>0,4 \geq a_{i}+t, a_{j}-t \geq 0} \alpha_{t}(-z)^{t} \mathcal{A}_{a_{1}, \ldots, a_{i}+t, \ldots, a_{j}-t, \ldots, a_{n}}(z)
$$

where the presence of positive powers of $z$ in the numerator makes only physical poles contribute to boundary values. Similar framework can be obtained for boundary contributions of theories with fermions, for which one example will be presented in next section. A direct implication of our result is that even with a bad deformation in gauge theory, boundary contributions are also cut-constructible in some sense, i.e, they can be obtained from pole contributions of some other related theories recursively.

\section{Examples}

In this section, we will use two simple examples to demonstrate our idea. We will discuss gluon MHV amplitude in detail as the first example and then briefly for another example.

\subsection{The first example: gluon MHV amplitude}

Since the purpose of this note is to understand boundary contributions of bad deformation, not to use it in practical calculations, we will illustrate our idea simply by using MHV amplitude of gluons, which is given by

$$
\mathcal{A}\left(s^{-}, n^{-}\right)=\frac{\langle s \mid n\rangle^{4}}{\langle 1 \mid 2\rangle\langle 2 \mid 3\rangle \ldots\langle n \mid 1\rangle} .
$$

We take the bad deformation $\left.\left\langle s^{-}\right| t^{+}\right]$with $t<s$. The $z$-dependence can be written down explicitly as

$$
\mathcal{A}_{t=0, s=4}=\frac{\langle s+z t \mid n\rangle^{4}}{\langle 1 \mid 2\rangle\langle 2 \mid 3\rangle \ldots\langle s-1 \mid s+z t\rangle\langle s+z t \mid s+1\rangle \ldots\langle n \mid 1\rangle},
$$

where for later convenience we have explicitly written the power of $\eta$-expansion at positions $t, s$ in the $\mathcal{N}=4$ super-amplitude. Splitting it into pole part of $z$ as well as divergent parts of $z^{a}, a \geq 0$, we obtain

following boundary values, i.e., the coefficient of $z^{0}$-term. If $t \neq s-1$ or $t \neq s+1$, the highest power of $z$ is $z^{2}$ and the coefficient of $z^{0}$ term is given by

$$
\begin{aligned}
\mathcal{B}_{t=0, s=4}= & \frac{\langle n \mid t\rangle^{2}}{\langle s-1 \mid t\rangle^{3}\langle t \mid s+1\rangle^{3}}\left(6\langle n \mid s\rangle^{2}\langle s-1 \mid t\rangle^{2}\langle t \mid s+1\rangle^{2}\right. \\
& -4\langle n \mid s\rangle\langle n \mid t\rangle\langle s-1 \mid t\rangle\langle t \mid s+1\rangle(\langle s \mid s+1\rangle\langle s-1 \mid t\rangle+\langle s-1 \mid s\rangle\langle t \mid s+1\rangle) \\
& \left.+\langle n \mid t\rangle^{2}\left(\langle s \mid s+1\rangle^{2}\langle s-1 \mid t\rangle^{2}+\langle s \mid s+1\rangle\langle s-1 \mid t\rangle\langle s \mid s-1\rangle\langle s+1 \mid t\rangle+\langle s \mid s-1\rangle^{2}\langle s+1 \mid t\rangle^{2}\right)\right) .
\end{aligned}
$$


If $t=s-1$ or $t=s+1$, the highest power becomes $z^{3}$, and the $z^{0}$ term would be (we just write down the case $t=s-1$ )

$$
\begin{aligned}
\mathcal{B}_{t=0, s=4}= & \frac{-\langle n \mid s-1\rangle}{\langle s \mid s-1\rangle\langle s-1 \mid s+1\rangle^{4}}\left(-\langle n \mid s-1\rangle^{3}\langle s \mid s+1\rangle^{3}-6\langle n \mid s\rangle^{2}\langle s-1 \mid s+1\rangle^{2}\langle n \mid s-1\rangle\langle s \mid s+1\rangle\right. \\
& \left.+4\langle n \mid s\rangle\langle s-1 \mid s+1\rangle\left(\langle n \mid s-1\rangle^{2}\langle s \mid s+1\rangle^{2}+\langle n \mid s\rangle^{2}\langle s-1 \mid s+1\rangle^{2}\right)\right) .
\end{aligned}
$$

It is worth to notice that the numerical coefficients of each term in expression (3.2) and (3.3) is 1, 4, 6, 4, 1, which are an indication of matter contents of $\mathcal{N}=4$ theory.

Now we apply our framework (2.8) to this example. The relevant combination can be easily found to be

$$
\oint \frac{d z}{z}\left(\mathcal{A}_{t=0, s=4}(z)-4 z \mathcal{A}_{t=1, s=3}(z)+6 z^{2} \mathcal{A}_{t=2, s=2}(z)-4 z^{3} \mathcal{A}_{t=3, s=1}(z)+z^{4} \mathcal{A}_{t=4, s=0}(z)\right)=0 .
$$

By using following results

$$
\begin{array}{ll}
\mathcal{A}_{t=4, s=0}=\frac{\langle t \mid n\rangle^{4}}{\langle 1 \mid 2\rangle\langle 2 \mid 3\rangle \ldots\langle n \mid 1\rangle}, & \mathcal{A}_{t=3, s=1}=\frac{\langle s \mid n\rangle\langle t \mid n\rangle^{3}}{\langle 1 \mid 2\rangle\langle 2 \mid 3\rangle \ldots\langle n \mid 1\rangle}, \\
\mathcal{A}_{t=2, s=2}=\frac{\langle s \mid n\rangle^{2}\langle t \mid n\rangle^{2}}{\langle 1 \mid 2\rangle\langle 2 \mid 3\rangle \ldots\langle n \mid 1\rangle}, & \mathcal{A}_{t=1, s=3}=\frac{\langle s \mid n\rangle^{3}\langle t \mid n\rangle}{\langle 1 \mid 2\rangle\langle 2 \mid 3\rangle \ldots\langle n \mid 1\rangle},
\end{array}
$$

the integrand of (3.4) becomes

$$
\frac{\langle s \mid n\rangle^{4}}{\langle 1 \mid 2\rangle\langle 2 \mid 3\rangle \ldots\langle s-1 \mid s+z t\rangle\langle s+z t \mid s+1\rangle \ldots\langle n \mid 1\rangle},
$$

which gives zero boundary contribution as it should be. Then we rewrite (3.4) as follows

$$
\begin{aligned}
\mathcal{B}_{t=0, s=4} & \equiv \oint \frac{d z}{z} \mathcal{A}_{t=0, s=4}(z) \\
& =-\oint \frac{d z}{z}\left(-4 z \mathcal{A}_{t=1, s=3}(z)+6 z^{2} \mathcal{A}_{t=2, s=2}(z)-4 z^{3} \mathcal{A}_{t=3, s=1}(z)+z^{4} \mathcal{A}_{t=4, s=0}(z)\right) .
\end{aligned}
$$

It is easy to see that one can use (3.7) to calculate boundary contributions in the first line. Since the $z$-factor appears in numerator, there is no pole contribution at $z=0$ in (3.7), and all contributions come from physical poles. To calculate these terms we can use formula

$$
\oint \frac{d z A(z)}{z} z^{k}=-\sum_{\alpha} A_{L}\left(z_{\alpha}\right) \frac{z_{\alpha}^{k}}{P^{2}} A_{R}\left(z_{\alpha}\right), \quad k \geq 1 .
$$

We will apply formula (3.8) to (3.7) and compare results with the one given by (3.2) and (3.3).

The case $t=s-1, n=s+1$ :

As a warm up let us start from the simplest case $t=s-1$ and $n=s+1$. There are four terms from recursion relation (3.7), as illustrated in figure (1). The first three diagrams do not contribute because the 

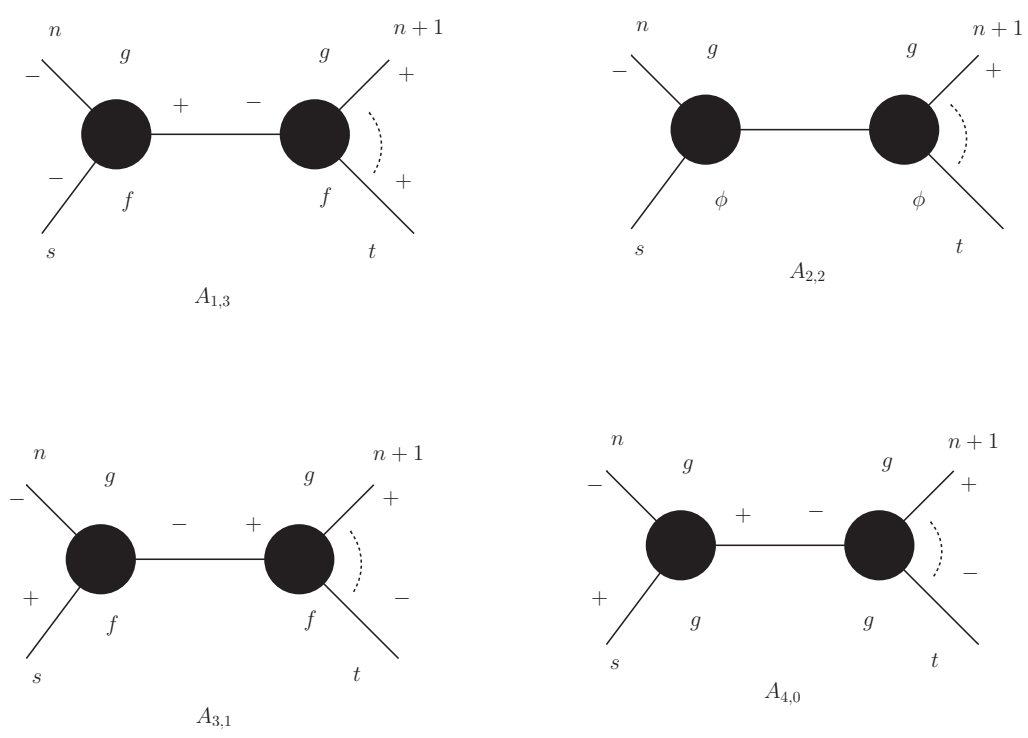

Figure 1: The four diagrams are these boundary contributions for $A_{0,4}$ with $t=s-1$ and $n=s+1$ using the recursion relation.

left hand side of each diagram is three-point MHV amplitude with all $\lambda$ proportional to each other, thus gives zero value. So boundary contributions of $A_{t=0, s=4}$ are given by $A_{L}^{4,0}\left(z_{\alpha}\right) \frac{z_{\alpha}^{4}}{P^{2}} A_{R}^{4,0}\left(z_{\alpha}\right)$. Using

$$
z_{\alpha}=-\frac{\langle n \mid s\rangle}{\langle n \mid t\rangle}
$$

we obtain

$$
\mathcal{B}_{t=0, s=4}=A_{L}^{4,0}\left(z_{\alpha}\right) \frac{z_{\alpha}^{4}}{P^{2}} A_{R}^{4,0}\left(z_{\alpha}\right)=\left(-\frac{\langle n \mid s\rangle}{\langle n \mid t\rangle}\right)^{4} A_{L}^{4,0}\left(z_{\alpha}\right) \frac{1}{P^{2}} A_{R}^{4,0}\left(z_{\alpha}\right)=\frac{\langle s \mid n\rangle^{4}}{\langle 1 \mid 2\rangle\langle 2 \mid 3\rangle \ldots\langle n \mid 1\rangle}=A_{0,4}(0)(3
$$

which agrees with the one given by (3.3).

The case $t=s-1, n \neq s+1$ :

The second case we are considering is $t=s-1$ and $n \neq s+1$. Again there are four terms from recursion relation (3.7), as shown in figure (2). All these four diagrams give nonzero boundary contributions to $A_{t=0, s=4}$. By using

$$
z_{\alpha}=-\frac{\langle s+1 \mid s\rangle}{\langle s+1 \mid t\rangle}
$$

we have

$$
\left|s\left(z_{\alpha}\right)\right\rangle=|\hat{s}\rangle=|s\rangle-\frac{\langle s+1 \mid s\rangle}{\langle s+1 \mid t\rangle}|t\rangle=-\frac{\langle t \mid s\rangle}{\langle s+1 \mid t\rangle}|s+1\rangle
$$



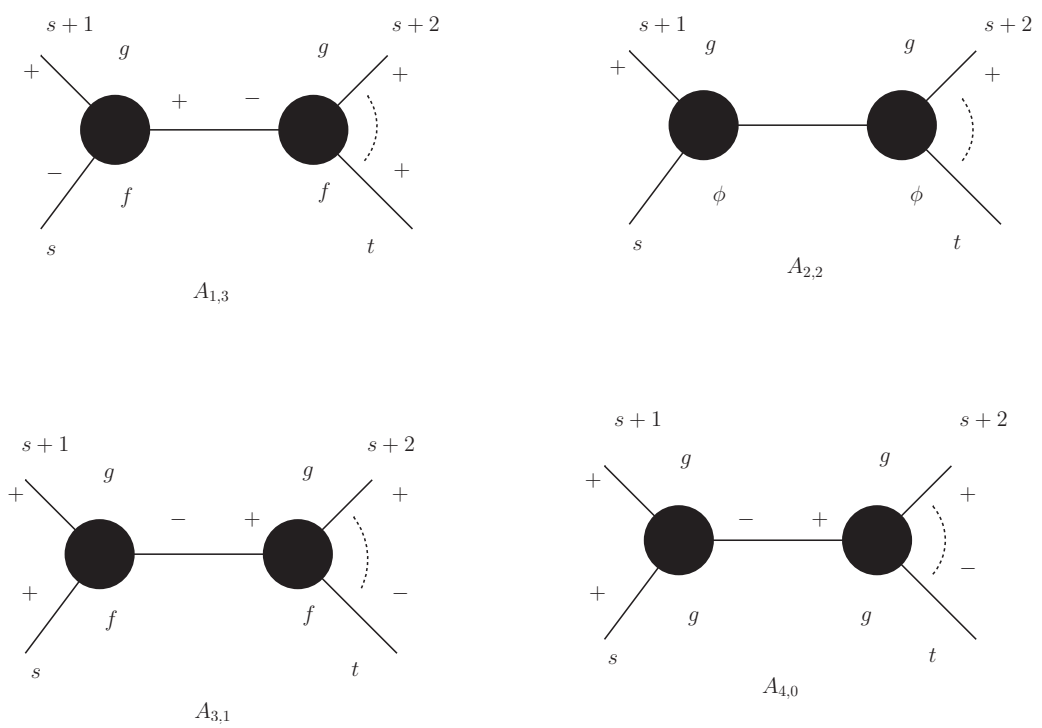

Figure 2: The four diagrams are these boundary contributions for $A_{0,4}$ with $t=s-1$ and $n \neq s+1$ using the recursion relation.

where we have used the Schouten identity. All contributions from four diagrams in figure (2) have common denominator as

$$
\begin{aligned}
& P(0)^{2}\langle\hat{t} \mid \hat{P}\rangle\langle\hat{P} \mid s+2\rangle\langle s+2 \mid s+3\rangle \cdots\langle t-1 \mid \hat{t}\rangle[\hat{s} \mid s+1][s+1 \mid-\hat{P}][-\hat{P} \mid \hat{s}] \\
= & {[s+1 \mid s]^{4}\langle s \mid s+1\rangle\langle s+1 \mid s+2\rangle \cdots\langle t-1 \mid t\rangle\langle t \mid s\rangle }
\end{aligned}
$$

and these four numerators are given separately as

$$
\begin{array}{ll}
A_{1,3}: & -4[\hat{s} \mid s+1][\hat{P} \mid s+1]^{3}\langle\hat{P} \mid n\rangle^{3}\langle\hat{t} \mid n\rangle z_{\alpha}=4 z_{\alpha}[\hat{s} \mid s+1]^{4}\langle n \mid \hat{s}\rangle^{3}\langle\hat{t} \mid n\rangle, \\
A_{2,2}: & {[\hat{s} \mid s+1]^{2}[\hat{P} \mid s+1]^{2}\langle\hat{P} \mid n\rangle^{2}\langle\hat{t} \mid n\rangle^{2} 6 z_{\alpha}^{2}=6 z_{\alpha}^{2}[\hat{s} \mid s+1]^{4}\langle n \mid \hat{s}\rangle^{2}\langle\hat{t} \mid n\rangle^{2}} \\
A_{3,1}: & -4 z_{\alpha}^{3}[\hat{s} \mid s+1]^{3}[\hat{P} \mid s+1]^{1}\langle\hat{P} \mid n\rangle^{1}\langle\hat{t} \mid n\rangle^{3}=4 z_{\alpha}^{3}[\hat{s} \mid s+1]^{4}\langle n \mid \hat{s}\rangle^{1}\langle\hat{t} \mid n\rangle^{3} \\
A_{4,0}: & z_{\alpha}^{4}[\hat{s} \mid s+1]^{4}\langle\hat{t} \mid n\rangle^{4}
\end{array}
$$

Sum up these four numerators we have

$$
[\hat{s} \mid s+1]^{4}\left[\langle n \mid \hat{s}\rangle+z_{\alpha}\langle\hat{t} \mid n\rangle\right]^{4}-[\hat{s} \mid s+1]^{4}\langle n \mid \hat{s}\rangle^{4} .
$$

Combining with the common denominator we get the final result

$$
\begin{aligned}
\mathcal{B}_{t=0, s=4} & =\frac{\left[\langle n \mid \hat{s}\rangle+z_{\alpha}\langle\hat{t} \mid n\rangle\right]^{4}-\langle n \mid \hat{s}\rangle^{4}}{\langle s \mid s+1\rangle\langle s+1 \mid s+2\rangle \cdots\langle t-1 \mid t\rangle\langle t \mid s\rangle} \\
& =\frac{\langle n \mid s\rangle^{4}-\left[\frac{\langle n \mid s+1\rangle\langle t \mid s\rangle}{\langle t \mid s+1\rangle}\right]^{4}}{\langle s \mid s+1\rangle\langle s+1 \mid s+2\rangle \cdots\langle t-1 \mid t\rangle\langle t \mid s\rangle}
\end{aligned}
$$



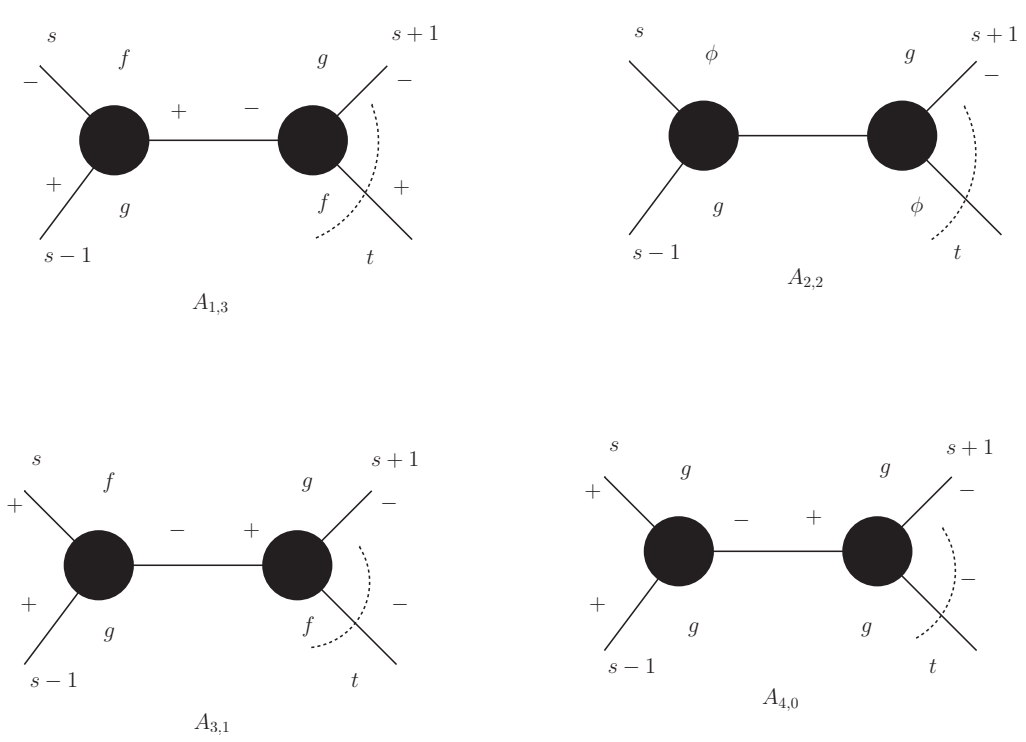

Figure 3: These four diagrams are the A part of boundary contributions for $A_{0,4}$ with $t \neq s-1, t \neq s+1$ and $n=s+1$ using the recursion relation.

where in the second step we have used (3.11) and (3.12). This result agrees with the one given by (3.3).

\section{Case $t \neq s-1, t \neq s+1$ and $\mathbf{n}=\mathbf{s}+\mathbf{1}$ :}

The third case we are considering is $t \neq s-1, t \neq s+1$ and $n=s+1$. There are five diagrams contributing to boundary values. We divide these five diagrams into two parts, as part A shown in figure (3) and part B, in figure (4). We first calculate contributions of part A from figure (3), where $z_{\alpha}^{A}$ is given by

$$
z_{\alpha}^{A}=-\frac{\langle s-1 \mid s\rangle}{\langle s-1 \mid t\rangle}
$$

The common denominator of these four contributions is

$$
\begin{aligned}
& P(0)^{2}\langle\hat{t} \mid \hat{P}\rangle\langle\hat{P} \mid s+1\rangle\langle s+1 \mid s+2\rangle \cdots\langle t-1 \mid \hat{t}\rangle[s-1 \mid \hat{s}][\hat{s} \mid \hat{P}][\hat{P} \mid s-1] \\
= & {[s-1 \mid s]^{4}\langle t \mid t+1\rangle \cdots\langle s-1 \mid s\rangle\langle\hat{s} \mid s+1\rangle\langle s+1 \mid s+2\rangle \cdots\langle t-1 \mid t\rangle . }
\end{aligned}
$$

By using Schouten identity, we have

$$
\langle\hat{s} \mid s+1\rangle=\frac{\langle s \mid t\rangle\langle s-1 \mid s+1\rangle}{\langle s-1 \mid t\rangle},
$$

thus the denominator becomes

$$
[s-1 \mid s]^{4} \frac{\langle s \mid t\rangle\langle s-1 \mid s+1\rangle}{\langle s-1 \mid t\rangle\langle s \mid s+1\rangle} \prod_{i}\langle i \mid i+1\rangle .
$$




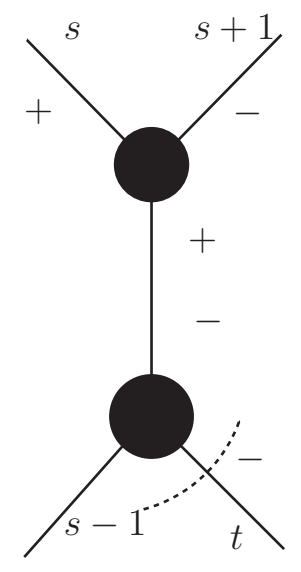

$A_{4,0}$

Figure 4: The diagram is the B part of boundary contributions for $A_{0,4}$ with $t \neq s-1, t \neq s+1$ and $n=s+1$ using the recursion relation.

By similar calculations for these four numerators as in (3.14) we get

$$
\begin{aligned}
& {[\hat{s} \mid s-1]^{4}\left[\langle n \mid \hat{s}\rangle+z_{\alpha}\langle t \mid n\rangle\right]^{4}-[\hat{s} \mid s-1]^{4}\langle n \mid \hat{s}\rangle^{4} } \\
= & {[\hat{s} \mid s-1]^{4}\langle n \mid s\rangle^{4}-[\hat{s} \mid s-1]^{4}\left[\frac{\langle n \mid s-1\rangle\langle s \mid t\rangle}{\langle s-1 \mid t\rangle}\right]^{4} . }
\end{aligned}
$$

Putting these results together we have part A contributions from figure (3)

$$
\frac{\langle s-1 \mid t\rangle\langle s \mid s+1\rangle}{\langle s \mid t\rangle\langle s-1 \mid s+1\rangle} \frac{1}{\prod_{i}\langle i \mid i+1\rangle}\left[\langle n \mid s\rangle^{4}-\left[\frac{\langle n \mid s-1\rangle\langle s \mid t\rangle}{\langle s-1 \mid t\rangle}\right]^{4}\right] .
$$

The part B contribution from figure (雨) can be calculated by similar way and the result is

$$
\frac{\langle s+1 \mid t\rangle\langle s-1 \mid s\rangle}{\langle s-1 \mid s+1\rangle\langle s \mid t\rangle} \frac{1}{\prod_{i}\langle i \mid i+1\rangle}\langle n \mid s\rangle^{4} .
$$

By using Schouten identity $\langle s-1 \mid t\rangle\langle s \mid s+1\rangle+\langle s+1 \mid t\rangle\langle s-1 \mid s\rangle=\langle s \mid t\rangle\langle s-1 \mid s+1\rangle$, we can combine part $\mathrm{A}$ and part $\mathrm{B}$ contributions together and get the final result for boundary values $A_{0,4}$ with $t \neq s-1$, $t \neq s+1$ and $\mathrm{n}=\mathrm{s}+1$ is

$$
\mathcal{B}_{t=0, s=4}=\frac{1}{\prod_{i}\langle i \mid i+1\rangle}\left[\langle n \mid s\rangle^{4}-\frac{\langle s-1 \mid t\rangle\langle s \mid s+1\rangle}{\langle s \mid t\rangle\langle s-1 \mid s+1\rangle}\left[\frac{\langle n \mid s-1\rangle\langle s \mid t\rangle}{\langle s-1 \mid t\rangle}\right]^{4}\right] .
$$

The result agrees with the one given by (3.2).

The case $t \neq s-1, t \neq s+1, n \neq s+1$ and $n \neq s-1$ :

The last case we are considering is $t \neq s-1, t \neq s+1, n \neq s+1$ and $n \neq s-1$. There are totally eight diagrams contributing to $A_{0,4}$, again we divide eight diagrams into two parts, as part $\mathrm{A}$ shown in figure (5) and part B, in figure (6). 

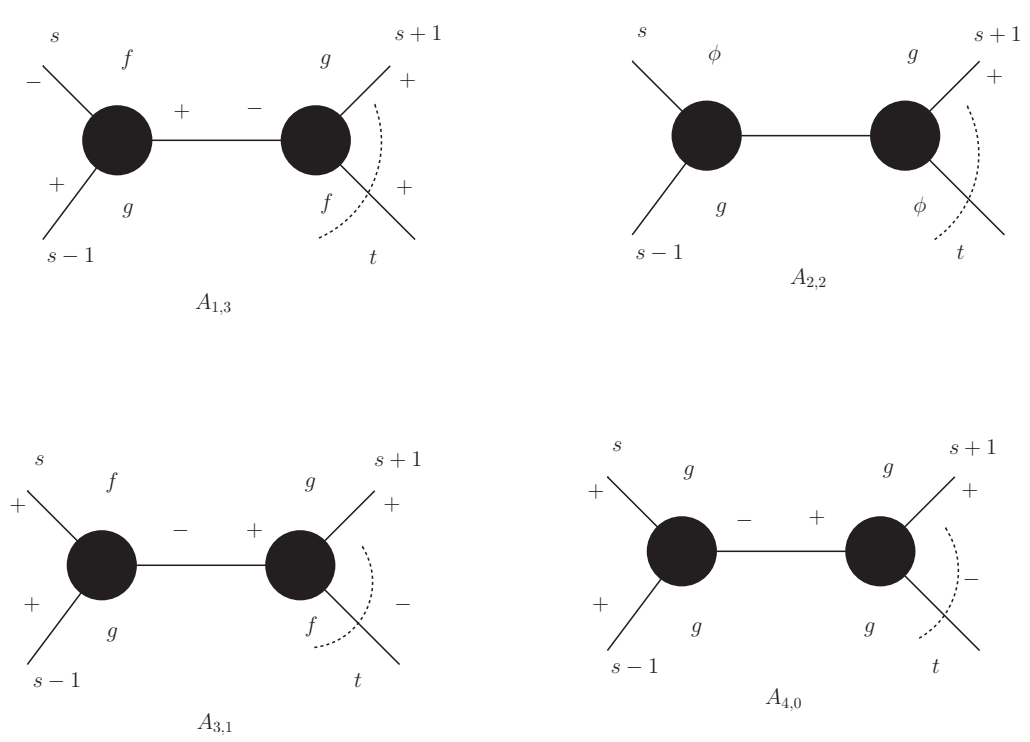

Figure 5: These diagrams are the A part of the boundary contributions for $A_{0,4}$ with $t \neq s-1, t \neq s+1, n \neq s+1$ and $n \neq s-1$ using the recursion relation.

By similar calculations as done in (3.16) we get results for part A in figure (5) as

$$
\frac{\langle s-1 \mid t\rangle\langle s \mid s+1\rangle}{\langle s \mid t\rangle\langle s-1 \mid s+1\rangle} \frac{1}{\prod_{i}\langle i \mid i+1\rangle}\left[\langle n \mid s\rangle^{4}-\left[\frac{\langle n \mid s-1\rangle\langle s \mid t\rangle}{\langle s-1 \mid t\rangle}\right]^{4}\right]
$$

and similarly the contribution for part B in figure (6) as

$$
\frac{\langle s+1 \mid t\rangle\langle s-1 \mid s\rangle}{\langle s-1 \mid s+1\rangle\langle s \mid t\rangle} \frac{1}{\prod_{i}\langle i \mid i+1\rangle}\left[\langle n \mid s\rangle^{4}-\left[\frac{\langle n \mid s+1\rangle\langle t \mid s\rangle}{\langle t \mid s+1\rangle}\right]^{4}\right] .
$$

So the final boundary value for $A_{0,4}$ with $t \neq s-1, t \neq s+1, n \neq s+1$ and $n \neq s-1$ is

$$
\begin{aligned}
\mathcal{B}_{t=0, s=4} & =\frac{1}{\prod_{i}\langle i \mid i+1\rangle}\left[\langle n \mid s\rangle^{4}-\frac{\langle s-1 \mid t\rangle\langle s \mid s+1\rangle}{\langle s \mid t\rangle\langle s-1 \mid s+1\rangle}\left[\frac{\langle n \mid s-1\rangle\langle s \mid t\rangle}{\langle s-1 \mid t\rangle}\right]^{4}\right. \\
& \left.-\frac{\langle s+1 \mid t\rangle\langle s-1 \mid s\rangle}{\langle s-1 \mid s+1\rangle\langle s \mid t\rangle}\left[\frac{\langle n \mid s+1\rangle\langle t \mid s\rangle}{\langle t \mid s+1\rangle}\right]^{4}\right] .
\end{aligned}
$$

The result agrees with the one given by (3.2).

\subsection{The second example: amplitude with two fermions and four gluons $\Lambda_{1}^{+} \Lambda_{2}^{-} g_{3}^{-} g_{4}^{-} g_{5}^{+} g_{6}^{+}$}

The $A\left(\Lambda_{1}^{+} \Lambda_{2}^{-} g_{3}^{-} g_{4}^{-} g_{5}^{+} g_{6}^{+}\right)$amplitude has been calculated in [21 by choosing a good deformation, here we will discuss boundary contributions with bad deformation for this one, thus provide a generalization of our discussions to cases besides gluons. We will consider two different bad deformations.

First we consider the bad shifting $\left.\left\langle 4^{-}\right| 5^{+}\right]$. With this choice, there is no contribution from physical poles and the whole amplitude is given by boundary contributions. There are four diagrams under $\left.\left\langle 4^{-}\right| 5^{+}\right]$ 

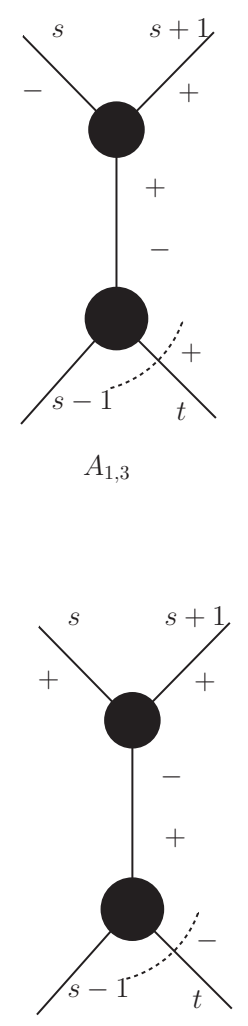

$A_{3,1}$

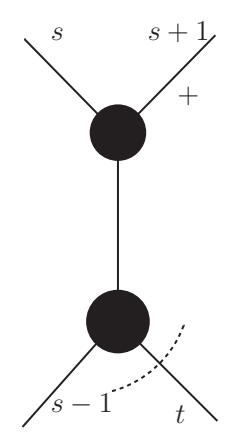

$A_{2,2}$

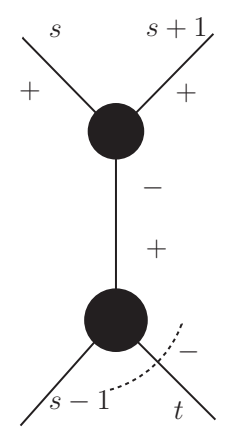

$A_{4,0}$

Figure 6: These diagrams are the $\mathrm{B}$ part of the boundary contributions for $A_{0,4}$ with $t \neq s-1, t \neq s+1, n \neq s+1$ and $n \neq s-1$ using the recursion relation.

deformation, as shown in figure (7). The recursion relation for boundary contributions is given by

$$
\begin{aligned}
\mathcal{B}_{t=0, s=4} & \equiv \oint \frac{d z}{z} \mathcal{A}_{t=0, s=4}(z) \\
& =-\oint \frac{d z}{z}\left(-z^{3} \mathcal{A}_{t=3, s=1}(z)+z^{4} \mathcal{A}_{t=4, s=0}(z)\right)
\end{aligned}
$$

according to the general formula (2.8). Compared to the one given by (3.7), we see that there are only two terms with numerator coefficient 1 . The reason is that this amplitude has fermions $\Lambda_{1}, \Lambda_{2}$, so in order to get non-zero contributions, $\Lambda$ must pair up with the same kind of fermions (recall that there are four different fermions in $\mathcal{N}=4$ SYM theory).

The boundary contributions from figure (7.a) and (7.c.) are given by

$$
\begin{aligned}
& -A_{L}^{3,1}\left(z_{a}\right) \frac{z_{a}^{3}}{P^{2}} A_{R}^{3,1}\left(z_{a}\right)+A_{L}^{4,0}\left(z_{c}\right) \frac{z_{c}^{4}}{P^{2}} A_{R}^{4,0}\left(z_{c}\right) \\
= & -\frac{s_{561}^{2}\langle 1|5+6| 2]}{\langle 5 \mid 6\rangle\langle 1 \mid 6\rangle[2 \mid 3][3 \mid 4]\langle 5|1+6| 2]\langle 1|5+6| 4]}
\end{aligned}
$$




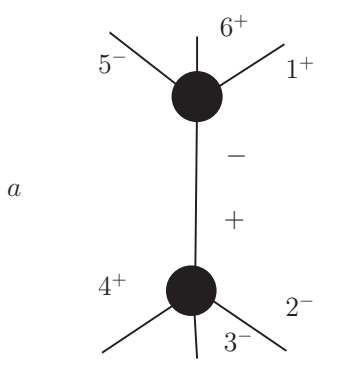

$A_{3,1}$
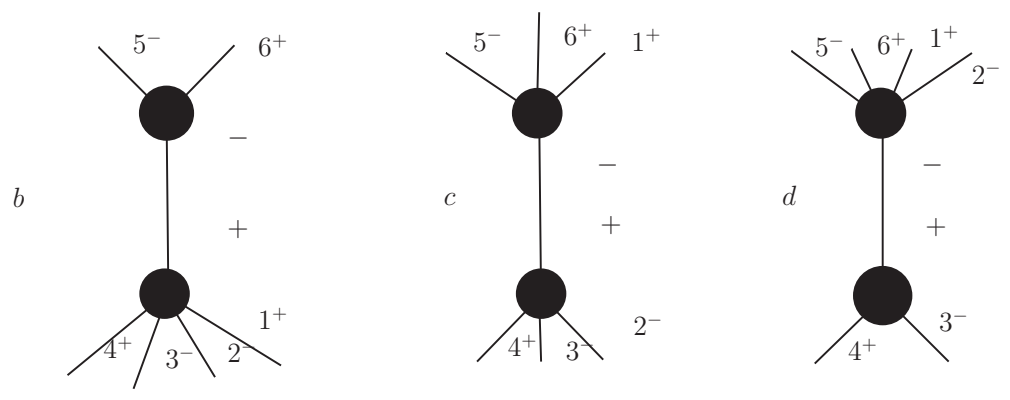

$A_{4,0}$

Figure 7: The boundary term for $A\left(\Lambda_{1}^{+} \Lambda_{2}^{-} g_{3}^{-} g_{4}^{-} g_{5}^{+} g_{6}^{+}\right)$with $\left.\left\langle 4^{-}\right| 5^{+}\right]$shifting using the recursion relation.

while the boundary contributions from figure (7.b) and figure (7.d) are given by

$$
-\frac{\langle 3 \mid 2\rangle^{2}\langle 3 \mid 1\rangle[5 \mid 6]^{3}}{s_{456}\langle 1 \mid 2\rangle[4 \mid 5]\langle 3|4+5| 6]\langle 1|5+6| 4]}-\frac{[6 \mid 1]^{2}[6 \mid 2]\langle 3 \mid 4\rangle^{3}}{s_{345}\langle 4 \mid 5\rangle[1 \mid 2]\langle 3|1+2| 6]\langle 5|1+6| 2]}
$$

where $s_{561}=\left(k_{5}+k_{6}+k 1\right)^{2}, s_{456}=\left(k_{4}+k_{5}+k_{6}\right)^{2}$ and $s_{345}=\left(k_{3}+k_{5}+k_{5}\right)^{2}$. Putting these four terms together, we find that

$$
\begin{aligned}
\mathcal{B}_{t=0, s=4} & =-\frac{s_{561}^{2}\langle 1|5+6| 2]}{\langle 5 \mid 6\rangle\langle 1 \mid 6\rangle[2 \mid 3][3 \mid 4]\langle 5|1+6| 2]\langle 1|5+6| 4]} \\
& -\frac{\langle 3 \mid 2\rangle^{2}\langle 3 \mid 1\rangle[5 \mid 6]^{3}}{s_{456}\langle 1 \mid 2\rangle[4 \mid 5]\langle 3|4+5| 6]\langle 1|5+6| 4]}-\frac{[6 \mid 1]^{2}[6 \mid 2]\langle 3 \mid 4\rangle^{3}}{s_{345}\langle 4 \mid 5\rangle[1 \mid 2]\langle 3|1+2| 6]\langle 5|1+6| 2]} \\
& =A\left(\Lambda_{1}^{+} \Lambda_{2}^{-} g_{3}^{-} g_{4}^{-} g_{5}^{+} g_{6}^{+}\right),
\end{aligned}
$$

where the last identity can be checked with the result obtained in [21].

Our general framework can also be used to solve the problem raised in [22], where the author concluded that one can not use nearby fermions to take BCFW deformation if the helicity configuration is $(+,-)$ or $(-,+)$. The author also stated that the pair of one fermion and an adjacent gluon with the same helicity is also not suitable for BCFW deformation. Now we understand that one can use nearby fermions to do BCFW deformation if one is able to find boundary contributions. Using the recursion relation, the 
boundary contributions with $\left.\left\langle 1^{+}\right| 2^{-}\right]$shifting are given by

$$
\mathcal{B}^{\prime}=\oint \frac{d z}{z} A_{1,3}(z)=\oint \frac{d z}{z} z^{3} A_{4,0}(z)=-A_{L}^{4,0}\left(z_{\alpha}\right) \frac{z_{\alpha}^{3}}{P^{2}} A_{R}^{4,0}\left(z_{\alpha}\right)
$$

Contributions from physical poles under this deformation is zero and the whole amplitude is again given by boundary contributions. There are three diagrams with pole contributions from $A_{4,0}$, i.e., the pure gluon helicity configuration $(-,+,-,-,+,+)$. These three terms are exactly the one given by (3.31).

\section{Conclusion and discussions}

In this paper we have studied boundary contributions with bad deformation in gauge theory. We deduce a very useful on-shell recursion relation to calculate the boundary contributions from $\mathcal{N}=4$ SUSY amplitudes. It provides useful understanding of bad deformations although we can always choose good deformations to calculate the gauge theory amplitudes. Especially our recursion relation shows the cutconstructibility of boundary contributions in generalized sense, i.e., they are given by pole contributions of related theory. Obviously same consideration can be done for amplitudes with gravitons.

\section{Acknowledgments}

BF is supported by fund from Qiu-Shi, the Fundamental Research Funds for the Central Universities with contract number 2009QNA3015, as well as Chinese NSF funding under contract No.10875104.

\section{References}

[1] Bo Feng, Junqi Wang, Yihong Wang and Zhibai Zhang, "BCFW Recursion Relation with Nonzero Boundary Contribution," JHEP 1001, 019 (2010) [arXiv:0911.0301 [hep-th]].

[2] R. Britto, F. Cachazo and B. Feng, Nucl. Phys. B 715, 499 (2005) [arXiv:hep-th/0412308].

[3] R. Britto, F. Cachazo, B. Feng and E. Witten, Phys. Rev. Lett. 94, 181602 (2005) [arXiv:hep-th/0501052].

[4] E. Witten, Commun. Math. Phys. 252, 189 (2004) [arXiv:hep-th/0312171].

[5] R. Britto, F. Cachazo and B. Feng, Nucl. Phys. B 725, 275 (2005) [arXiv:hep-th/0412103].

[6] N. Arkani-Hamed, F. Cachazo, C. Cheung and J. Kaplan, JHEP 1003, 020 (2010) [arXiv:0907.5418 [hep-th]].

[7] N. Arkani-Hamed, F. Cachazo and C. Cheung, JHEP 1003, 036 (2010) [arXiv:0909.0483 [hep-th]].

[8] N. Arkani-Hamed, J. Bourjaily, F. Cachazo and J. Trnka, arXiv:0912.3249 [hep-th].

[9] N. Arkani-Hamed, J. Bourjaily, F. Cachazo and J. Trnka, arXiv:0912.4912 [hep-th].

[10] R. H. Boels, arXiv:1003.2989 [hep-th].

[11] Paolo. Benincasa, Freddy. Cachazo, "Consistancy Conditions On The S-Matix Of Massless Particles" arXiv:0705.4305[hep-th]. 
[12] N. Arkani-Hamed and J. Kaplan, JHEP 0804, 076 (2008) [arXiv:0801.2385 [hep-th]].

[13] D. Vaman and Y. P. Yao, JHEP 0604, 030 (2006) [arXiv:hep-th/0512031].

[14] P. D. Draggiotis, R. H. P. Kleiss, A. Lazopoulos and C. G. Papadopoulos, Eur. Phys. J. C 46, 741 (2006) [arXiv:hep-ph/0511288].

[15] P. Benincasa, C. Boucher-Veronneau and F. Cachazo, JHEP 0711, 057 (2007) [arXiv:hep-th/0702032].

[16] C. Cheung, arXiv:0808.0504 [hep-th].

[17] V. P. Nair, Phys. Lett. B 214, 215 (1988).

[18] N. Arkani-Hamed, F. Cachazo and J. Kaplan, arXiv:0808.1446 [hep-th].

[19] J.M.Drummond, J.Henn, G.P.Korchemsky and E.Sokatchev, "Dual superconformal symmetry of scattering amplitudes in $\mathcal{N}=4$ super-Yang Mills theory," Nucl. Phys. B 828(2010)317 [arXiv:0807.1095 [hep-th]].

[20] A. Brandhuber, P. Heslop and G. Travaglini, Phys. Rev. D 78, 125005 (2008) [arXiv:0807.4097 [hep-th]].

[21] Ming-xing Luo and Congkao Wen, "Recursion relations for Tree Amplitudes in Super Gauge Theories," JHEP 0503, 004 (2005) [arXiv:hep-th/0501121].

[22] Ming-xing Luo and Congkao Wen, "Compact Formulas for Tree Amplitudes of Six Partions," Phys.Rev.D 71: 091501 (2005) [arXiv:hep-th/0502009]. 